\title{
Torsade de pointes caused by polypharmacy and substance abuse in a patient with human immunodeficiency virus
}

\author{
Jane M. Prosser • Angela Mills • \\ Eugene S. Rhim • Jeanmarie Perrone
}

Received: 12 February 2008 / Accepted: 20 July 2008 / Published online: 22 August 2008

(C) Springer-Verlag London Ltd 2008

\begin{abstract}
Drug-induced QT prolongation is a potentially dangerous adverse effect of some medication combinations. When QT prolongation progresses to torsade de pointes, life-threatening or fatal outcomes may result. A 57-year-old man with a history of human immunodeficiency syndrome on abacavir, nevirapine, tenofovir, voriconazole, and methadone presented to the emergency department with a chief complaint of new-onset seizures. The physical exam was unremarkable. The electrocardiogram demonstrated sinus bradycardia and a prolonged $\mathrm{QT}_{\mathrm{c}}$ interval of 690 $\mathrm{ms}$. In the emergency department, he had several episodes of torsade de pointes (TdP) and ventricular tachycardia that resolved spontaneously. These episodes were accompanied by an alteration in mentation and generalized twitching. Magnesium and amiodarone were effective in terminating the dysrhythmia. The patient had multiple risk factors for
\end{abstract}

J. M. Prosser $(\bowtie)$

Departments of Emergency Medicine and Toxicology,

New York City Poison Control,

New York University Hospital,

455 First Avenue Suite \# 123,

New York, NY 10016, USA

e-mail: jprosser100@gmail.com

A. Mills · J. Perrone

Department of Emergency Medicine,

Hospital of the University of Pennsylvania,

Ground Ravdin,

Philadelphia, PA 14103, USA

E. S. Rhim

Manhattan Cardiac Arrhythmia Associates,

10 East 86th Street Suite 502,

New York, NY 10028, USA prolonged QT syndrome including human immunodeficiency virus infection, methadone therapy, and polypharmacy leading to potential drug interactions. Physicians must be aware of multidrug interactions potentiating QT prolongation and leading to torsade de pointes.

Keywords Torsade de pointes $\cdot$ Methadone .

Human immunodeficiency virus · Cocaine · Voriconazole

\section{Introduction}

Torsade de pointes (TdP) is a life-threatening dysrhythmia that can result from long QT syndrome. Many medications and medical conditions cause acquired long QT syndrome. We present a case of TdP caused by a prolonged QT associated with polypharmacy and substance abuse in a patient with human immunodeficiency virus (HIV) infection. Methadone is the most widely used medication worldwide for drug replacement therapy in illicit drug users according to the World Health Organization [1]. The global incidence of HIV is estimated to be 33 million [2]. It is important that physicians be aware of possible complications of interactions between these common elements.

\section{Case report}

A 57-year-old male with HIV (CD4 ${ }^{+}$count 339/ $\mu$ l, viral load unknown), right lower extremity amputation, retroorbital aspergillosis, and a history of intravenous drug abuse presented to the emergency department with a chief complaint of seizures. The patient reported two episodes of 
muscle twitching and urinary incontinence without loss of consciousness. He reported numerous episodes of palpitations and shortness of breath over the past several days. He denied weakness, syncope, fever, dizziness, chest pain, and postictal periods. Two days prior he had been started on highly active antiretroviral therapy with abacavir (600 $\mathrm{mg} \mathrm{qd}$ ), nevirapine (200 $\mathrm{mg} \mathrm{qd}$ ), and tenofovir (300 $\mathrm{mg} \mathrm{qd}$ ). His medication regimen also included voriconazole (200 $\mathrm{mg}$ b.i.d.) for a retro-orbital aspergillosis infection diagnosed 6 months prior and methadone $(125 \mathrm{mg} /$ day $)$ for detoxification of heroin addiction. He reported current use of tobacco, marijuana and alcohol, and remote cocaine use.

Initial vital signs were temperature $98.0^{\circ} \mathrm{F}$, pulse 60 beats per minute (bpm), blood pressure 126/61 mmHg, respiratory rate 20 breaths per minute, and oxygen saturation $98 \%$ without supplemental oxygen. He was awake, alert, and oriented. Notable findings on physical exam were a $3 / 6$ holosystolic murmur and right lower extremity amputation. The rest of his exam was unremarkable.

Cardiac monitoring revealed bradycardia with heart rates ranging from 30 to $60 \mathrm{bpm}$. Frequent polymorphic premature ventricular beats and nonsustained ventricular tachycardia were present. The electrocardiogram (ECG) showed sinus rhythm at a rate of $79 \mathrm{bpm}$ with a right bundle branch block and a left anterior fascicular block
(Fig. 1). The QT interval was prolonged. The corrected QT interval $\left(\mathrm{QT}_{\mathrm{c}}\right.$ using Bazett's formula) was $690 \mathrm{~ms}$. A baseline ECG from 5 months prior revealed sinus bradycardia at a rate of $51 \mathrm{bpm}$, similar bundle branch blocks, and a $\mathrm{QT}_{\mathrm{c}}$ of $440 \mathrm{~ms}$.

Laboratory results showed an unremarkable complete blood count and serum chemistries with a potassium of $3.7 \mathrm{mmol} / \mathrm{l}$ (reference $3.5-5.3 \mathrm{mmol} / \mathrm{l}$ ), a magnesium of $0.9 \mathrm{mmol} / \mathrm{l}(0.53-1.03 \mathrm{mmol} / \mathrm{l})$, and calcium of $2.15 \mathrm{mmol} / \mathrm{l}(2.12-2.62 \mathrm{mmol} / \mathrm{l})$. Troponin I was indeterminate at $0.7 \mu \mathrm{g} / \mathrm{l}$. Head computed tomography scan was unremarkable. Chest X-ray revealed mild pulmonary edema with cardiomegaly.

While awaiting admission, the patient experienced several episodes of TdP and ventricular tachycardia. Each episode lasted 1-2 min and resolved spontaneously. During these episodes the patient was unable to communicate verbally, but did not lose consciousness. He exhibited myoclonic muscle twitching and was incontinent of urine. These episodes were similar to those he had experienced earlier in the day. Treatment was started with $2 \mathrm{~g}$ of magnesium. A bolus of amiodarone was given, followed by a continuous infusion. Once therapy was started, the patient had no additional episodes.

In the cardiac care unit, overdrive pacing was initiated. An isoproterenol infusion was titrated to maintain a heart

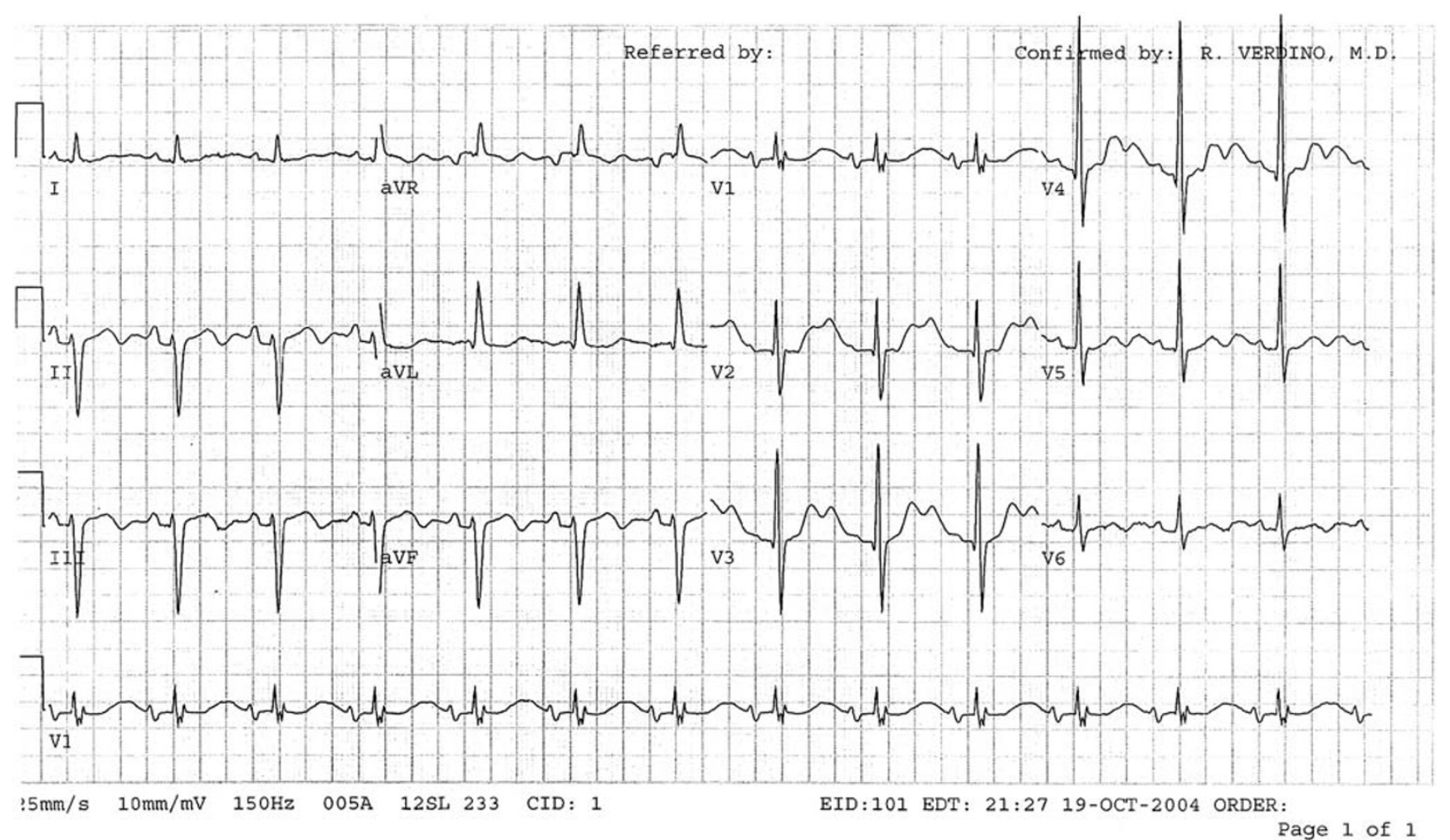

Fig. 1 ECG 
rate of $90 \mathrm{bpm}$ to prevent reoccurrence of TdP. The patient was continued on methadone to prevent withdrawal; voriconazole and his antiretroviral medications were held. Urine drug screening was positive for marijuana and cocaine. A pacemaker/implantable cardioverter defibrillator (ICD) was placed. After the placement of the ICD, voriconazole was restarted.

He was discharged 12 days after admission with resolution of his symptoms but persistent $\mathrm{QT}_{\mathrm{c}}$ prolongation. The patient was maintained on the same medications that he was taking prior to presentation. He was scheduled to taper his methadone dose in the hope of discontinuing methadone completely. Interrogation of the ICD 1 month after discharge revealed no further episodes of TdP.

\section{Discussion}

There are two types of long QT syndrome: congenital and acquired. Myocardial repolarization abnormalities lead to QT prolongation on the surface ECG. This leads to an increased risk of polymorphic ventricular tachycardia known as torsade de pointes (TdP). Congenital causes are secondary to genetic abnormalities of myocardial ion channels [3]. Acquired long QT syndrome has many causes including medication, HIV, intracranial pathology, electrolyte abnormalities, and renal and hepatic failure. Drugs cause most cases of acquired long QT by inhibiting delayed rectifier potassium channels, leading to prolonged myocardial repolarization [4]. The most common inciting drugs are antidysrhythmics and psychiatric drugs [5]. Illicit drugs such as cocaine are another cause. The two forms of long QT overlap. Patients with subclinical or unrecognized long QT are at increased risk for development of TdP when started on medications that prolong the QT interval.

The QT segment is considered abnormal when the $\mathrm{QT}_{\mathrm{c}}$ is $>440 \mathrm{~ms}$ in males or $>460 \mathrm{~ms}$ in females. Drug-induced $\mathrm{TdP}$ is most likely to occur with a $25 \%$ increase in the baseline QT interval or if the $\mathrm{QT}_{\mathrm{c}}$ is $>500 \mathrm{~ms}$. Greater than $90 \%$ of $\mathrm{TdP}$ occurs when the $\mathrm{QT}_{\mathrm{c}}$ is $>500 \mathrm{~ms}$ [4]. The development of TdP occurs when an early afterdepolarization follows a long RR interval. An early afterdepolarization is an impulse that occurs before the myocardial cell has repolarized, during phase 2 or 3 of the action potential. This leads to abnormal dispersion of myocardial repolarization which can cause the development of a reentrant circuit. This is the reason that $\mathrm{TdP}$ in acquired long QT syndrome is referred to as pause-dependent ventricular tachycardia. Bradycardia is therefore a risk factor for the development of TdP. The QT interval becomes longer as the heart rate decreases. TdP is usually self-terminating but can lead to sustained ventricular tachycardia and death [6].
This patient had at least four risk factors for QT prolongation. These included HIV infection, cocaine use, and treatment with methadone and voriconazole. HIV infection has been shown to be an independent risk factor for QT prolongation. Kocheril et al. showed a prevalence of a prolonged QT in 28.6\% of hospitalized HIV patients compared with $7.0 \%$ of hospitalized patients without HIV infection [7]. Although the pathophysiology behind this is unclear, hypotheses include myocarditis and autonomic neuropathies [8].

Cocaine blocks the delayed rectifier potassium channel and has been shown to lengthen the QT interval [5, 9]. Cocaine use may have been the precipitating factor causing torsade in this patient. It is difficult to ascertain the time course as he refused to acknowledge recent cocaine use despite positive urine toxicology.

Many studies have shown that methadone increases the QT interval and may lead to TdP [10-14]. As a result, a black box warning for methadone was issued in 2006 by the US Food and Drug Administration, alerting practitioners to the increased risk of TdP particularly in patients with drug interactions. High-dose methadone regimens may place patients at increased risk of TdP. In animal models, methadone has been shown to affect the action potential by decreasing the rate of depolarization and increasing the action potential duration. It has also been shown to have negative chronotropic effects that may lead to bradycardia $[10,14]$. Maremmani et al. found that $83 \%$ of patients in a methadone maintenance program had QT prolongation [11]. Additionally, patients on methadone are at higher risk for TdP when given other QT-prolonging drugs or when hypokalemic [12]. A methadone level was not obtained. Little is known about the correlation between an elevated methadone level and the onset of cardiac toxicity.

Voriconazole has been reported to cause QT prolongation and TdP. Voriconazole is a potent blocker of the P450 system, leading to increased concentrations of drugs metabolized by this system. It specifically inhibits the CYP2C19, CYP2C9, and CYP3A4 enzymes that metabolize methadone. The maximum plasma concentration of methadone can be increased by $65 \%$. The prescribing information states that voriconazole in combination with methadone may lead to increased plasma concentrations of methadone and QT prolongation. Careful monitoring is recommended as a reduced dose of methadone may be necessary [15].

Some protease inhibitors have been shown to prolong the QT interval; however, this patient was not on a protease inhibitor and QT prolongation from other antiretrovirals has not been reported.

Treatment of acquired QT syndrome consists of removal of the offending agents. Electrolyte abnormalities should be corrected. Serum potassium should be maintained between 
4.5 and $5.0 \mathrm{mmol} / 1$. Magnesium is the first-line therapy; $2 \mathrm{~g}$ of magnesium may be given and repeated if necessary. Overdrive pacing and isoproterenol can be titrated to keep the heart rate $>90 \mathrm{bpm}$. This will shorten the QT interval. Implantable cardioverter defibrillator devices do not decrease the number of arrhythmias, but can be lifesaving if events occur [4]. Antidysrythmics may also be used. In this patient, amiodarone was given following the advanced cardiac life support protocol for ventricular tachycardia in a patient with a pulse. There is little guidance in the literature to suggest which antidysrhythmic is the most effective agent in drug-induced TdP. Consideration should be given to the metabolism of amiodarone by CYP3A4 and also its risk of $\mathrm{QT}_{\mathrm{c}}$ prolongation, though the incidence of associated TdP is less than $1 \%$ [16].

\section{Conclusion}

HIV infection and substance abuse are global problems. Methadone maintenance is the most commonly used drug replacement therapy for opioid dependence worldwide. Patients with HIV and substance abuse are likely to have other comorbidities and may be on multiple medications. Methadone-associated TdP in the setting of methadone therapy often occurs with the addition of another QTprolonging agent. Physicians must be aware of QT prolongation secondary to methadone therapy and the drug interactions that may potentiate toxicity from methadone. Torsade de pointes should be included in the differential diagnosis when evaluating patients on methadone, especially those complaining of syncope or seizures. Practitioners should be aware of associated risk factors for QT prolongation in these patients including concomitant HIV infection or cocaine use.

\section{Conflicts of interest None.}

\section{References}

1. World Health Organization (2004) Policy brief: reduction of HIV transmission through drug-dependence treatment. Available via: http://www.who.int/hiv/pub/advocacy/en/drugdependencetreat menten.pdf. Accessed 12 Feb 2008

2. Global Health Facts (2007) People living with HIV/AIDS. Available via: http://www.globalhealthfacts.org/topic.jsp? $i=1 \&$ gclid $=\mathrm{CN}$ XowtLhvpECFQFIHgod2k7syQ. Accessed 12 Feb 2008

3. Moss AJ (2003) Long QT syndrome. JAMA 289:2041-2053

4. Gowda RM, Khan IA, Wilbur SL et al (2004) Torsade de pointes: the clinical considerations. Int J Cardiol 96:1-6

5. Singh N, Singh HK, Singh PP, Khan IA (2001) Cocaine-induced torsades de pointes in idiopathic long Q-T syndrome. Am J Ther 8:299-302

6. Al-Khatib SM, LaPointe NM, Kramer JM, Califf RM (2003) What clinicians should know about the QT interval. JAMA 289:2120-2127

7. Kocheril AG, Bokhari SA, Batsford WP et al (1997) Long $\mathrm{QT}_{\mathrm{c}}$ and torsades de pointes in human immunodeficiency virus disease. Pacing Clin Electrophysiol 20:2810-2818

8. Villa A, Foresti V, Confalonieri F (1995) Autonomic neuropathy and prolongation of the QT interval in human immunodeficiency virus infection. Clin Auton Res 5:48-52

9. Gamouras GA, Monir G, Plunkitt K et al (2000) Cocaine abuse: repolarization abnormalities and ventricular arrhythmias. Am J Med Sci 320:9-12

10. Gil M, Sala M, Anguera I et al (2003) QT prolongation and torsades de pointes in patients infected with human immunodeficiency virus and treated with methadone. Am J Cardiol 92:995-997

11. Maremmani I, Pacini M, Cesaroni $C$ et al (2005) $\mathrm{QT}_{\mathrm{c}}$ interval prolongation in patients on long-term methadone maintenance therapy. Eur Addict Res 11:44-49

12. Krantz MJ, Lewkowiez L, Hays H et al (2002) Torsade de pointes associated with very-high-dose methadone. Ann Intern Med 137:501-504

13. Kornick CA, Kilborn MJ, Santiago-Palma J et al (2003) $\mathrm{QT}_{\mathrm{c}}$ interval prolongation associated with intravenous methadone. Pain 105:499-506

14. Martell BA, Arnsten JH, Krantz M, Gourevitch MN (2005) The impact of methadone treatment on cardiac repolarization and conduction in opioid users. Am J Cardiol 95:915-918

15. Prescribing information Vfend. Available via: http://www.pfizer. com/pfizer/download/uspi vfend.pdf. Accessed 5 Feb 2008

16. Hohnloser SH, Klingenheben T, Singh BH (1994) Amiodaroneassociated proarrhythmic effects. A review with special reference to torsade de pointes tachycardia. Ann Intern Med 121:529-533 